\title{
Efficient, Effective, and Insightful Tackling of the High-Dose-Rate Brachytherapy Treatment Planning Problem for Prostate Cancer using Evolutionary Multi-Objective Optimization Algorithms
}

\author{
Ngoc Hoang Luong \\ Centrum Wiskunde \& Informatica \\ Amsterdam, The Netherlands \\ Hoang.Luong@cwi.nl \\ Yury Niatsetski \\ Elekta \\ Veenendaal, The Netherlands \\ yury.niatsetski@elekta.com
}

\author{
Anton Bouter \\ Centrum Wiskunde \& Informatica \\ Amsterdam, The Netherlands \\ Anton.Bouter@cwi.nl \\ Cees Witteveen \\ Delft University of Technology \\ Delft, The Netherlands \\ C.Witteveen@tudelft.nl
}

\author{
Marjolein C. van der Meer \\ Academic Medical Center \\ Amsterdam, The Netherlands \\ marjolein.vandermeer@amc.uva.nl \\ Arjan Bel \\ Academic Medical Center \\ Amsterdam, The Netherlands \\ A.Bel@amc.uva.nl
}

\author{
Tanja Alderliesten \\ Academic Medical Center \\ Amsterdam, The Netherlands \\ T.Alderliesten@amc.uva.nl
}

\author{
Peter A.N. Bosman \\ Centrum Wiskunde \& Informatica \\ Amsterdam, The Netherlands \\ Peter.Bosman@cwi.nl
}

\begin{abstract}
We address the problem of high-dose-rate brachytherapy treatment planning for prostate cancer. The problem involves determining a treatment plan consisting of the so-called dwell times that a radiation source resides at different positions inside the patient such that the prostate volume and the seminal vesicles are covered by the prescribed radiation dose level as much as possible while the organs at risk, e.g., bladder, rectum, and urethra, are irradiated as little as possible. The problem is highly constrained, following clinical requirements for radiation dose distribution while the planning process for treatment planners to design a clinically-acceptable treatment plan is strictly time-limited. In this paper, we propose that the problem can be formulated as a bi-objective optimization problem that intuitively describes trade-offs between target volumes to be radiated and organs to be spared. We solve this problem with the recently-introduced Multi-Objective Real-Valued Genepool Optimal Mixing Evolutionary Algorithm (MO-RV-GOMEA), which is a promising MOEA that is able to effectively exploit dependencies between problem variables to tackle complicated problems in the continuous domain. MO-RV-GOMEA also has the capability to perform partial evaluations if problem structures allow local variations in existing solutions to be efficiently computed, substantially accelerating the overall optimization performance. Experiments on real medical data and comparison with state-of-theart MOEAs confirm our claims.
\end{abstract}

Permission to make digital or hard copies of all or part of this work for personal or classroom use is granted without fee provided that copies are not made or distributed for profit or commercial advantage and that copies bear this notice and the full citation on the first page. Copyrights for components of this work owned by others than ACM must be honored. Abstracting with credit is permitted. To copy otherwise, or republish, to post on servers or to redistribute to lists, requires prior specific permission and/or a fee. Request permissions from permissions@acm.org.

GECCO '17 Companion, Berlin, Germany

(C) 2017 ACM. 978-1-4503-4939-0/17/07 ..\$15.00

DOI: http://dx.doi.org/10.1145/3067695.3082491

\section{CCS CONCEPTS}

-Mathematics of computing $\rightarrow$ Evolutionary algorithms;

\section{KEYWORDS}

linkage learning, multi-objective optimization, partial evaluation, brachytherapy, radiotherapy, cancer treatment planning

ACM Reference format:

Ngoc Hoang Luong, Anton Bouter, Marjolein C. van der Meer, Yury Niatsetski, Cees Witteveen, Arjan Bel, Tanja Alderliesten, and Peter A.N. Bosman. 2017. Efficient, Effective, and Insightful Tackling of the High-DoseRate Brachytherapy Treatment Planning Problem for Prostate Cancer using Evolutionary Multi-Objective Optimization Algorithms. In Proceedings Companion of GECCO '17 Companion, Berlin, Germany, fuly 15-19, 2017, 8 pages.

DOI: http://dx.doi.org/10.1145/3067695.3082491

\section{INTRODUCTION}

Radiotherapy is a frequently used form of treatment for cancer. Brachytherapy (BT) [14] is a form of internal radiotherapy where radiation sources are placed inside, or passed through, the patient's body, close to the tumors, as opposed to the External Beam Radiation Therapy (EBRT) where radiation is directed at the tumors from outside the patient's body. A potential advantage of BT is that due to its local nature, the radioactive dose distribution can be shaped to conform to the shape of the treatment volume better, and healthy surrounding organs/tissues can thus be spared from undesired radiation risks. Furthermore, the most common way to apply BT, the so-called High-Dose-Rate (HDR) BT, is by using a radioactive source with a high strength, resulting in fewer radiation treatment fractions. In this paper, we focus on HDR-BT treatment for prostate cancer but the methodology can be straightforwardly adapted to other types of BT involving radioactive sources with 
a lower strength. To deliver radiation sources to the target volumes (i.e., the prostate, and in some cases, the seminal vesicles), normally, 14-20 catheters (depending on the prostate size) are inserted into patient's body through the transperineal skin. These catheters are connected to a device, called the afterloader, which controls the movement of radiation sources through the catheters. Each catheter has a certain number of $d$ well positions where the source can pause to release radiation for a certain amount of time, termed dwell time, before moving to the next position. The longer the dwell time at a dwell position, the more radioactive dose is distributed to the surrounding volume. The list of all dwell times at all dwell positions comprises an HDR-BT treatment plan. Making a clinically-acceptable BT plan is not a trivial task, and involves many clinical requirements that need to be satisfied. On the one hand, dwell times should be long enough to cover the target volumes as much as possible with a certain prescribed radiation dose, effectively sterilizing cancer cells. On the other hand, dwell times should be as short as possible to keep the radiation delivered to normal tissues and other nearby Organs At Risk (OARs), i.e., urethra, rectum, bladder, under certain clinically-acceptable upper bounds. Aiming to cover the target volumes while sparing OARs, BT treatment planning is intrinsically a multi-objective optimization problem, where a single utopian solution (i.e., a treatment plan) that optimizes all objectives at the same time does not exist. Instead, there exists the Pareto-optimal set of non-dominated solutions, that are optimal in the sense that improving any objective of these solutions deteriorates their other objectives. The image set of the Pareto-optimal set in the objective space forms the so-called Pareto-optimal front that exhibits the possible trade-offs between the involved objectives. The goal then in multi-objective optimization is to find a so-called approximation set of solutions that is as close as possible to the optimal Pareto set of solutions, often measured in the objective space.

Available BT treatment planning software packages, see e.g., [5, $7,10]$, however, do not tackle the problem in a true multi-objective manner. Instead, all the objectives following from the clinical requirements are combined into a single optimization function by the weighted-sum approach, obtaining thus a single solution that corresponds with each setting of the weighting coefficient vector. The proper setting of these weights can hardly be determined $a$ priori because it involves taking into account the geometry of the patient's organs, the configuration of the inserted catheters, and the preferences of the treating physician. Weighted-sum approaches with some rule-of-thumb coefficient settings often return treatment plans that do not match the trade-off that individual radiation oncologists prefer for specific patients. Therefore, BT treatment planners (i.e., radiation oncologists, radiation therapy technologists, and clinical physicists) often need to spend time to manually adjust the treatment plan until satisfied, sometimes up to an hour. In this paper, we tackle BT treatment planning in the true multiobjective optimization manner, approximating the set of optimal trade-offs between target volumes coverage versus OARs sparing. Such an approximation set explicitly quantifies and exhibits the compromises that need to be made. Treatment planners can make use of the obtained approximation set as a decision support tool to quickly locate the desired trade-off solution, which can be further adjusted, to arrive at a final plan.
The HDR-BT treatment procedure is time-constrained, where the planning task should normally be finalized within one hour. Optimization algorithms need to spend a certain number of iterations including treatment plan evaluations before acceptable plans can be obtained. BT treatment plan evaluation is a time-consuming operation, which involves calculations of the radiation dose distribution at target volumes and OARs. In this paper, we present how the dependency structure of dwell positions can be used to enhance the efficiency in generating promising candidate treatment plans. Such dependency knowledge can be acquired online by performing linkage learning during the optimization process or can be computed offline based on the geometry information of the implanted catheters. Always performing a full evaluation of treatment plans is unnecessary, especially if the offspring solutions differ from parent solutions at only a few dwell times. Therefore, the solving time can be substantially improved if local changes of treatment plans can be computed. To this end, we use the recentlyintroduced Multi-Objective Real-Valued Gene-pool Optimal Mixing Evolutionary Algorithm (MO-RV-GOMEA) [2], which has been shown to have superior performance on benchmark problems due to its capabilities of exploiting linkage information and performing partial evaluations in the context of continuous optimization. Here, we aim to see the impact of the advantages that MO-RVGOMEA has to offer in solving the BT treatment planning problem compared to the well-known Multi-Objective Evolutionary Algorithm (MOEA) NSGA-II [4] and the Multi-Objective Estimation-ofDistribution Algorithm (MOEDA) MAMaLGaM [1].

\section{PROBLEM MODELING}

\subsection{Clinical Practice}

An HDR-BT treatment for prostate cancer starts with the insertion of a number of catheters through the area between the patient's scrotum and anus (i.e., the perineum) aimed at target volumes (i.e., the prostate and seminal vesicles). The implanted catheters (i.e., implant) are firmly fixed to avoid displacements. Medical images (e.g., Computed Tomography (CT) or Magnetic Resonance Imaging (MRI) scans) of the patient's pelvic area are then acquired to be employed in the following treatment planning session. Treatment planners first draw the contours of the treatment targets and OARs on the obtained CT/MRI scans in BT treatment planning software. The inserted catheters are also delineated. Each catheter is discretized into a number of dwell positions where radiation sources can dwell, normally by a step size of $2.5 \mathrm{~mm}$ beginning from the first dwell position which is offset $5.0 \mathrm{~mm}$ from the tip of the catheter. To ensure the treatment targets are sufficiently treated while sparing OARs from radiation risks, only dwell positions within the target volumes expanded with a margin of $5.0 \mathrm{~mm}$ are activated. Dwell positions outside these volumes are kept inactive, which means they will not be considered when the treatment plan is made. The longer the time a source dwells at an active position (i.e., dwell time), the more radiation is delivered to surrounding tissues. A certain dose level is prescribed by radiation oncologists which is deemed sufficient to sterilize tumor cells for the specific tumor type. Dwell times should then be configured such that the entire prostate should receive at least the prescribed dose, 
Efficient, Effective, and Insightful Tackling of the High-Dose-Rate Brachytherapy Treatment Planning Problem for Prostate Cancer using Evolutionary Multi-Objective Optimization Algorithms

GECCO '17 Companion, July 15-19, 2017, Berlin, Germany

but not too much in order to allow healthy cells, which are less susceptible to radiation than tumor cells, to recover from being radiated. On the one hand, treatment plans with very long dwell times can kill all tumor tissues but can also cause undesirable damage to healthy tissues (i.e., necrosis, which is considered life-threatening). On the other hand, treatment plans with too short dwell times can spare healthy tissues, but insufficiently-treated tumor cells can still grow, making the whole treatment ineffective. BT treatment planners need to make a proper treatment plan that satisfies general clinical requirements as well as special criteria for each specific case. The approved plan is then used to treat the patient. The afterloader, which is connected to the inserted catheters, controls the movements of the radiation source through the catheters such that the source stays at each dwell position for the amount of time as indicated in the approved plan.

\subsection{Dose Distribution Evaluation}

Each treatment plan (i.e., a specific configuration of dwell times) brings about a dose distribution in the surrounding tissues. A plan is deemed clinically acceptable if its dose distribution satisfies the clinical requirements. A utopian plan that radiates all tumor cells with the prescribed dose while delivering no radiation to OARs never exists. Clinical requirements therefore often indicate the sufficient lower bounds of radiating target volumes and allowable upper bounds of radiating OARs. A widely-used set of clinical requirements, termed Dose-Volume (DV) $V_{x}^{o}$ criteria (or requirements), specify how large the cumulative volume of an organ $o$ receiving at least the radiation dose level $x$ should be. For example, it is often recommended that $V_{100}^{\text {prostate }} \geq 95 \%$, i.e., at least $95 \%$ of the prostate volume should be covered by $100 \%$ of the prescribed dose [6]. To treat tumor cells possibly existing in the vesicles, the requirement $V_{80}^{\text {vesicles }} \geq 95 \%$ can be employed to indicate that at least $95 \%$ of the volume of the vesicles should receive $80 \%$ of the prescribed dose. To prevent hot spots forming inside the prostate (i.e., the part of prostate that is radiated too much), it is required that $V_{200}^{\text {prostate }} \leq 20 \%$, i.e., not more than $20 \%$ of the prostate volume should be covered by $200 \%$ of the prescribed dose. Similarly, to protect OARs from radiation risks, there are DV criteria for each organ that set the upper bounds of the radiation levels that can be allowed to be delivered to that organ. For example, $V_{78}^{\text {rectum }} \leq 1 \mathrm{~cm}^{3}$, i.e., the rectum volume covered by $78 \%$ of the prescribed dose should not exceed 1 cubic centimeter $\left(\mathrm{cm}^{3}\right)$, or $V_{74}^{\text {bladder }} \leq 2 \mathrm{~cm}^{3}$, i.e., the bladder volume receiving at least $74 \%$ of the prescribed dose should not be more than $2 \mathrm{~cm}^{3}$. Table 1 presents the DV indices and their corresponding requirements currently employed at the Academic Medical Center (AMC), the hospital involved in this study. $t f$ It is impossible to calculate the radiation received by every sin-

\begin{tabular}{|c|c|c|c|c|}
\hline Prostate & Bladder & Rectum & Urethra & Vesicles \\
\hline$V_{100} \geq 95 \%$ & $V_{86} \leq 1 \mathrm{~cm}^{3}$ & $V_{78} \leq 1 \mathrm{~cm}^{3}$ & $V_{110} \leq 0.1 \mathrm{~cm}^{3}$ & $V_{80} \geq 95 \%$ \\
\hline$V_{150} \leq 50 \%$ & $V_{74} \leq 2 \mathrm{~cm}^{3}$ & $V_{74} \leq 2 \mathrm{~cm}^{3}$ & & \\
\hline$V_{200} \leq 20 \%$ & & & & \\
\hline
\end{tabular}

Table 1: BT treatment planning DV criteria at AMC.

gle cell because the number of cells in an organ is prohibitively large. Therefore, DV index values are often approximated by evaluating the radiation at a certain number of so-called dose calculation points. The strength of the employed radiation source and the relative position between a dwell position and a dose calculation point determine the dose rate that indicates the amount of dose irradiated from the source when it resides at that dwell position to the dose calculation point per second (i.e., Gy/s). Dose rates can be computed based on the TG-43 protocol (the American Association of Physicists in Medicine AAPM Task Group No. 43 Report) [12]. The total radiation at each dose calculation point is the combined dose delivered from all the active dwell positions corresponding to the dwell times of the treatment plan. Let $D$ be the set of all dose calculation points, $|D|=n_{D}$. Let $T$ be the set of all dwell positions, $|T|=n_{T}$. With a certain source strength, $R$ is an $n_{D} \times n_{T}$ matrix where $\boldsymbol{R}_{i j}$ indicates the dose rate associated with dwell position $j$ and dose calculation point $i$. Let $\boldsymbol{t}$ be the vector of dwell times at all active dwell positions (i.e., $\boldsymbol{t}$ is a treatment plan). The vector $\boldsymbol{d}$ of the amounts of radiation received at all dose calculation points can be computed as:

$$
\boldsymbol{d}=\boldsymbol{R} \boldsymbol{t}
$$

The vector $\boldsymbol{d}$ can be seen as representing the dose distribution associated with the treatment plan $\boldsymbol{t}$, from which the DV indices (in Table 1) can be approximated. The dose calculation points can be uniformly randomly generated inside each organ. Let $D_{o}$ be the set of all dose calculation points inside organ $o, D_{o} \subset D$. The DV index $V_{x}^{o}$ in relative terms (\%) can then be computed as:

$$
V_{x}^{o}=\frac{1}{\left|D_{o}\right|} \sum_{i \in D_{o}} \chi\left(d_{i}, x\right)
$$

where $d_{i}$ is the total amount of radiation received at the dose calculation point $i$, and $\chi\left(d_{i}, x\right)$ is an indicator function:

$$
\chi\left(d_{i}, x\right)= \begin{cases}1 & d_{i} \geq x \\ 0 & d_{i}<x\end{cases}
$$

The DV index $V_{x}^{o}$ in absolute terms $\left(\mathrm{cm}^{3}\right)$ can be straightforwardly computed by multiplying the result from Equation 2 with the total volume of the organ $o$. The more dose calculation points are used, the more accurate the approximation of the true DV indices is (disregarding uncertainties of delineation). However, using a large number of dose calculation points incurs a substantial amount of computing time, which slows down the planning process.

\subsection{Optimization Constraints}

BT treatment planners normally start the planning process from an initial plan, which is generated by BT treatment planning software $[7,10]$. Because of the difficulty in directly optimizing DV indices due to their discrete nature (Equation 2), planning software often solves simpler optimization models of the problem. Therefore, DV requirements (in Table 1) are not always satisfied by the proposed plans. Planners need to identify the causes of the violated requirements and then manually adjust the plan in a local manner (i.e., changing the values of some dwell times). For example, dwell times at dwell positions inside the under-radiated part of the prostate volume can be increased if $V_{100}^{\text {prostate }}<95 \%$. If the plan causes many hot spots, then very long dwell times can be decreased to reduce $V_{200}^{\text {prostate }}$. Planners often improve DV indices one by one, giving priority to the most violated criteria. Making a DV index satisfy its requirement, however, can deteriorate other indices and might make them violate their requirements. For example, increasing $V_{100}^{\text {prost }}$ 
can create new hot spots (i.e., large values of $V_{200}^{\text {prostate }}$ ) and cause other DV indices for OARs to exceed their recommended thresholds. How good the best possible treatment plan is depends on the quality of the implant (i.e., how well the catheters are inserted) and also on the geometry of the surrounding organs. When OARs are too close to target volumes, it is difficult to achieve good DV index values for both target coverage and OARs sparing at the same time. If the catheters are not inserted deep enough, it might be impossible to obtain $V_{100}^{\text {prostate }} \geq 95 \%$ without violating $V_{200}^{\text {prostate }} \leq 20 \%$. In these situations, BT treatment planners need to compromise. To allow treatment plans that violate some part of the clinical requirements to some degree to be obtained in the optimization process, we relax the thresholds of the DV requirements to enlarge the feasible search space. Specifically, the upper bounds of organ sparing indices are increased four times. For example, $V_{74}^{\text {bladder }} \leq 2 \mathrm{~cm}^{3}$ in the original clinical requirement becomes $V_{74}^{\text {bladder }} \leq 8 \mathrm{~cm}^{3}$. The lower bounds of target coverage indices are also decreased in a similar manner, e.g., $V_{100}^{\text {prostate }} \geq 95 \%$ becomes $V_{100}^{\text {prostate }} \geq 80 \%$. These relaxed requirements, presented in Table 2, are the constraints in our optimization model.

\begin{tabular}{|c|c|c|c|c|}
\hline Prostate & Bladder & Rectum & Urethra & Vesicles \\
\hline$V_{100} \geq 80 \%$ & $V_{86} \leq 4 \mathrm{~cm}^{3}$ & $V_{78} \leq 4 \mathrm{~cm}^{3}$ & $V_{110} \leq 0.4 \mathrm{~cm}^{3}$ & $V_{80} \geq 80 \%$ \\
\hline$V_{150}$ free & $V_{74} \leq 8 \mathrm{~cm}^{3}$ & $V_{74} \leq 8 \mathrm{~cm}^{3}$ & & \\
\hline$V_{200} \leq 80 \%$ & & & & \\
\hline
\end{tabular}

Table 2: DV criteria from Table 1 with relaxed feasibility thresholds. Because of the relaxation factor, $V_{150}^{\text {prostate }}$ has become unconstrained.

\subsection{Multi-Objective Optimization}

BT treatment planning is a multi-objective optimization problem, where each DV index criterion can be considered to be an optimization objective. Optimizing target coverage indices (i.e., $V_{100}^{\text {prostate }}$, $V_{80}^{\text {vesicles }}$ ) equals making them as large as possible (above the lower bound thresholds) while optimizing organ sparing indices (the remaining indices in Table 1) equals making them as small as possible (under the upper bound thresholds). These two groups of DV indices are conflicting with each other. Each treatment plan can be seen as a trade-off between all the involved indices. Of key interest is to find the set of optimal trade-offs, termed non-dominated solutions, where improving any DV index in one group worsens DV indices in the other group. It is beneficial for the planners to be informed about these best possible alternatives before approving a plan to be used for treating the patient.

However, optimizing all DV indices of Table 2 as separate objectives would result in an 8-objective optimization problem, which is difficult to be efficiently solved. Moreover, its resulting set of 8-dimensional trade-offs is also complicated to be visualized and interpreted. On the other hand, simply summing all indices of a group into an objective is also not favorable since this also reduces the insight into key information about DV indices, namely the minimally achieved levels. We therefore propose a bi-objective optimization model that retains important insights in trade-offs between DV indices. The two objectives are: the Least Coverage Index (LCI), which corresponds to the worst-scored DV index in the target coverage group, and the Least Safe Index (LSI), which corresponds to the worst-scored DV index in the organ sparing group.
For a candidate treatment plan $\boldsymbol{t}$, its LCI value can be considered as:

$$
L C I(\boldsymbol{t})=\min \left\{V_{100}^{\text {prostate }}, V_{80}^{\text {vesicles }}\right\}
$$

The value of LCI, that we would like to maximize, is $\in[0,1]$. The value 0 indicates that one target volume (i.e., prostate or seminal vesicles) has no dose calculation point covered by the recommended dose level for that target volume. The ideal value 1 indicates that the whole volume of each treatment target is covered by the recommended dose level. The value 0.95 indicates that both clinical requirements $V_{100}^{\text {prostate }} \geq 95 \%$ and $V_{80}^{\text {vesicles }} \geq 95 \%$ in Table 1 are satisfied.

Let $V_{x}^{o, \max }$ be the upper bound threshold for the dose level $x$ to the organ $o$ in Table 2. The further the value of DV index $V_{x}^{o}$ is below this threshold, the better the organ $o$ is spared from radiation risk. The distance of an organ sparing index $V_{x}^{o}$ value below the threshold $V_{x}^{o, \max }$ can be measured and normalized as:

$$
\delta\left(V_{x}^{o}\right)=1-\frac{V_{x}^{o}}{V_{x}^{o, \max }}
$$

The LSI of a candidate treatment plan $\boldsymbol{t}$ can then be defined as:

$$
\begin{array}{r}
\operatorname{LSI}(\boldsymbol{t})=\min \left\{\delta\left(V_{150}^{\text {prostate }}\right), \delta\left(V_{200}^{\text {prostate }}\right), \delta\left(V_{86}^{\text {bladder }}\right), \delta\left(V_{74}^{\text {bladder }}\right),\right. \\
\left.\delta\left(V_{78}^{\text {rectum }}\right), \delta\left(V_{74}^{\text {rectum }}\right), \delta\left(V_{110}^{\text {urethra }}\right)\right\}
\end{array}
$$

The value of LSI, that we would like to maximize, is $\in[0,1]$. The value 0 indicates that one organ sparing index $V_{x}^{o}$ is currently at its (relaxed) threshold $V_{x}^{o, \max }$. The ideal value 1 indicates that there is no dose calculation point in any organ receiving more radiation than its relaxed threshold dose level. Since the upper bounds of all organ sparing indices are increased (i.e., relaxed) by a factor of 4 , the value LSI $=0.75$ ensures that all clinical requirements for organ sparing indices in Table 1 are met.

The objective values of any candidate treatment plan can thus be evaluated on these two objectives (LCI, LSI), where it can be inferred that the values of all the other DV indices in a group are at least as good as the representative index of that group. A treatment plan satisfies all original clinical requirements in Table 1 if its LCI $\geq 0.95$ and LSI $\geq 0.75$. We further argue that this model bears resemblance with the treatment planning process in practice in the sense that planners often try to iteratively improve the most violated DV index.

\section{MO-RV-GOMEA}

MO-RV-GOMEA [2] maintains a population of potential solutions on which selection and variation are performed. Encountered nondominated solutions are stored in an adaptive elitist archive [11]. A key strength of MO-RV-GOMEA is the main variation operator that was first introduced for the discrete GOMEA [13], and was later adapted to the domain of real-valued variables [2]. This variation operator was shown to be able to successfully exploit linkage structure in a wide range of optimization problems [2,13].

Each generation of MO-RV-GOMEA starts with the selection phase, where the selection is based on the ranking of solutions after non-domination sorting [4]. The selection is then partitioned into a set of clusters, which will allow different directions of optimization in different parts of the Pareto front. For each objective of interest, this set of clusters contains one so-called single-objective cluster 
Efficient, Effective, and Insightful Tackling of the High-Dose-Rate Brachytherapy Treatment Planning Problem for Prostate Cancer using Evolutionary Multi-Objective Optimization Algorithms

GECCO '17 Companion, July 15-19, 2017, Berlin, Germany

for which the selection procedure only considers the respective objective. Apart from the selection, each solution in the population is also assigned to a nearby cluster, because the Gaussian model of one specific cluster is used in the variation of the solution.

A linkage model is used to explicitly define dependencies between subsets of variables. Each linkage model consists of a number of linkage sets, where each linkage set describes a subset of variables that are considered to be dependent. The linkage tree is a specific hierarchical linkage model that models a range of dependencies ranging from low-level to high-level dependencies. A linkage tree can be learned during optimization, in which case it is learned anew at the start of each generation. The linkage tree is initialized as a set of $\ell$ leaves of univariate problem indices. New nodes are added to the tree by iteratively merging the two nonmerged nodes that are considered the most dependent by a similarity metric, for which we use the mutual information metric, which is derived from the sample Pearson correlation coefficient [8]. Alternatively, a similarity metric can be defined based on the structure of the optimization problem, leading to a linkage tree that is fixed throughout all generations. Merging nodes continues until the root of the tree has been created, which naturally contains the indices of all problem variables. Each node of the linkage tree then defines one linkage set of the linkage model.

The parameters for variation are estimated as follows. For each linkage set of each cluster, a multivariate Gaussian probability distribution is estimated with maximum likelihood based on the selection. The probability distribution of a certain linkage set covers only the variables that are included in this linkage set. A distribution multiplier is also maintained for each linkage set, which allows the dynamic adaptation of the size of each Gaussian kernel based on the observed direction of improvement. Per linkage set of each cluster, the variation operation is applied to each solution in this cluster by sampling new values from the Gaussian distribution for the problem variables described by the respective linkage set, and inserting these into the solution. A fraction of the solutions in each cluster is shifted by the application of the Anticipated Mean Shift (AMS), which shifts parameters by a factor relative to the generational difference of means. The modification of the solution is then evaluated, but only maintained if this modification is considered to be an improvement. Otherwise, the modification is not accepted and the solution is returned to its previous state.

We refer the interested reader to [2] for a full description of $\mathrm{MO}$ RV-GOMEA.

\section{PERFORMANCE ENHANCEMENT}

\subsection{Exploiting Geometry Information}

MO-RV-GOMEA performs variations based on linkage models that indicate which problem variables (i.e., in this case, dwell times at dwell positions) exhibit some degree of dependency and should thus be treated together. In the context of black-box optimization, linkage models can be learned from the population. Although even the most comprehensive linkage model employed by MO-RVGOMEA, i.e., the linkage tree, can be efficiently learned, performing linkage learning in every cluster for each generation can incur some computing time overhead. It could be beneficial and more efficient if sufficient problem-specific information is available to construct the linkage models offline. For the BT treatment planning problem, it can be argued that dwell positions that are close to each other have stronger interactions than dwell positions that are far apart. Dwell times at neighboring dwell positions, therefore, should be treated together when performing variation. The coordinates of all active dwell positions, which are determined from the CT/MRI scans of the patients, can be used to compute the Euclidean distances between all pairs of dwell positions. Such geometry information can be directly used as a distance/similarity metric to construct the linkage tree as in Section 3. This offlineconstructed Euclidean-distance-based linkage tree can be employed during the optimization process without the need of online linkage learning in each cluster, potentially improving the effectiveness in creating promising candidate treatment plans.

\subsection{Partial Evaluations}

(MO-RV-)GOMEA differs from other evolutionary algorithms in its genetic-local-search-like variation operator that transforms each existing (parent) solution into a new (offspring) solution in a stepwise manner. At each step, a few problem variables' values (i.e., dwell times in this context) are altered, and the changes are only accepted if they improve the quality of the current solution (i.e., the candidate treatment plan at hand). Which problem variables are varied at each step is often determined by the linkages described by the employed linkage model such that variables having some degree of dependency should be jointly treated. In black-box optimization, each partially-altered solution needs to be fully evaluated to check for improvements. If the problem is sufficiently understood, the impact of such local changes can be efficiently computed/approximated using partial evaluations. The evaluation of a treatment plan involves the computing of the radiation dose received at each dose calculation point (see Equation 1) and the computing of DV indices (see Equation 2). The former takes a more substantial amount of computing time. The matrix-vector multiplication in Equation 1, however, does not need to be calculated completely anew when the vector of dwell times is changed at only a few elements (i.e., dwell positions). More specifically, the impact of such changes on the dose distribution can be efficiently computed by invoking only the columns of the dose-rate matrix $\boldsymbol{R}$ corresponding with the dwell positions where the dwell times are altered. Let $\boldsymbol{t}$ be an evaluated treatment plan with the corresponding dose distribution $\boldsymbol{d}$. Let $\boldsymbol{t}^{\prime}$ be a treatment plan that differs from $\boldsymbol{t}$ at only a few dwell positions, i.e., $\boldsymbol{t}^{\prime}=\boldsymbol{t}+\Delta \boldsymbol{t}$, in which $\Delta t$ has many zero elements. The dose distribution $\boldsymbol{d}^{\prime}$ associated with $\boldsymbol{t}^{\prime}$ can be computed as:

$$
d^{\prime}=R t^{\prime}=R(t+\Delta t)=R t+R \Delta t=d+R \Delta t
$$

where the $i$-th index of $R \Delta t$ can be computed as:

$$
(\boldsymbol{R} \Delta \boldsymbol{t})_{i}=\sum_{\substack{j=1 \\ \Delta t_{j} \neq 0}}^{n_{T}} \boldsymbol{R}_{i j} \Delta t_{j}
$$

which thus only involves the multiplication of the column $R_{* j}$ of $R$ with the corresponding non-zero elements $\Delta t_{j}$ of $\Delta t$. Note that in the implementation an entire vector $\Delta t$ is not explicitly computed, but rather only the compact vector of non-zero elements in $\Delta t$ corresponding to a linkage set in the linkage tree is computed. 
The step-wise operation of the variation operator of MO-RVGOMEA makes it straightforward for partial evaluations to be employed, if possible. For other existing MOEAs, such as MAMaLGaM or NSGA-II, each time a whole offspring solution is created at once, making partial evaluations impossible.

\section{EXPERIMENTS}

\subsection{Experiment Settings}

DICOM files of three anonymized HDR-BT cases for prostate cancer from the Academic Medical Center (AMC) were available for conducting our experiments. DICOM files are loaded into the BT treatment planning software Oncentra Brachy, from which the contours of the involved organs (i.e., prostate, seminal vesicles, bladder, rectum, and urethra) and the inserted catheter information can be exported. This extracted information is then used as the input data for our multi-objective optimization algorithm. Similar to clinical practice, we activate all dwell positions inside the two target volumes: the prostate and seminal vesicles, each with an extended margin of $5 \mathrm{~mm}$ while dwell positions within $1 \mathrm{~mm}$ margin of the urethra should be kept inactive. In each organ, we uniformly randomly generate 4,000 points, i.e., a total of 20,000 dose calculation points are employed each time. Such a number of random points is deemed sufficient for the purpose of performing optimization [9].

We perform experiments with three MO-RV-GOMEA variants employing three linkage models: the Univariate Factorization (UF) model where all dwell times are deemed independent from each other, the Linkage Tree (LT) model which is learned from the population in each generation, and the fixed LT which is constructed a priori based on the geometry information of active dwell positions. For each MO-RV-GOMEA we run two settings: 1) full treatment plan evaluations are always carried out to assess candidate treatment plans, and 2) partial evaluations are enabled to assess partially-altered treatment plans when performing solution variation. For the purpose of performance comparison between MO-RV-GOMEA and state-of-the-art MOEAs, we consider NSGAII [4] and MAMaLGaM [1]. NSGA-II employs the Simulated Binary Crossover (SBX $[3,4]$ ) operator to create offspring solutions in realvalued optimization. MAMaLGaM estimates a Gaussian mixture distribution over the $35 \%$ best solutions in the population and samples the learned distribution to generate offspring solutions in each generation. To eliminate the tuning of the population size parameter, for all algorithms, we implement the interleaved multistart scheme as introduced in [2]. For every patient case, we run each algorithm 30 times independently. Each optimization run is allowed to operate 1 hour to obtain an approximation set of non-dominated plans.

We use the hypervolume [15] to compare the performance of MOEAs. The hypervolume can be intuitively defined as the volume (or area in the case of bi-objective optimization) in the objective space that is covered by a set of non-dominated solutions and a reference point, which is a point that can be selected such that it will be dominated by any possible solutions. Here, since the range of the two objectives LCI and LSI is $[0,1]$, we can simply choose $(-0.1,-0.1)$ as the reference point.

\subsection{Results}

Figure 1 shows the graphs of hypervolume development along the running time (in seconds) averaged over 30 runs of each optimization algorithm for the three patient cases. To support some of our observations from the figure, we use the Mann-Whitney-Wilcoxon statistical hypothesis test for equality of medians with $p<0.05$ to see whether the final result obtained by one algorithm is statistically different from that of another algorithm. When partial evaluations are not enabled, i.e., MO-RV-GOMEA carries out a normal full evaluation every time a candidate solution needs to be assessed, all MO-RV-GOMEA variants are outperformed by both NSGA-II and MAMaLGaM. Using totally full evaluations is inefficient for MO-RV-GOMEA because treatment plan evaluation is a computationally expensive operation and the algorithm most often only modifies an existing solution in a few variables at each step before fully constructing a new (offspring) solution, which differs from other MOEAs like NSGA-II and MAMaLGaM that generate a whole new solution at once. While the slopes of the hypervolume development graphs of NSGA-II and MAMaLGaM flatten out at the end of the optimization runs, suggesting that both algorithms nearly converge, the ones of MO-RV-GOMEA variants still steepen up, indicating that they are still in the middle of the search. Note that using different linkage models that capture only higher-order dependencies of a certain minimum degree could have a substantial impact here and make the difference much smaller. However, using partial evaluations, as is possible here, could make such search for highly suitable linkage structures superfluous.

Indeed, when partial evaluations are enabled, all variants of MORV-GOMEA are substantially accelerated, outperforming both MAMaLGaM and NSGA-II in the cases of patients 2 and 3. At the termination time of one hour, in all three cases, MO-RV-GOMEA with the fixed LT and partial evaluations is the best algorithm, obtaining Pareto fronts with the highest hypervolume values, which are found to be statistically significantly different from the other MO-RV-GOMEA variants and the other MOEAs. Therefore, we consider the fixed LT with partial evaluations as the most suitable configuration for MO-RV-GOMEA to tackle the BT treatment planning problem and we only consider this configuration in the following discussion.

Figure 2 shows the Pareto fronts of non-dominated plans obtained by NSGA-II, MAMaLGaM, and MO-RV-GOMEA for the 3 cases. Each Pareto front is the combination of the 30 approximation sets obtained at the end of the 30 optimization runs of each algorithm. Our formulations of the two objectives LCI and LSI (see Section 2.4) imply that treatment plans satisfying all clinical requirements (in Table 1) should equal to, or (Pareto-)dominate, the point $(0.95,0.75)$. Graphically, clinically-acceptable treatment plans can be located in the top-right corner of the graphs between $(0.95,0.75)$ and $(1,1)$. Pareto fronts shown in Figure 2 indicate that treatment plans that satisfy all clinical requirements are achievable in all three cases. The Pareto fronts obtained by MO-RV-GOMEA are always better than the ones obtained by NSGA-II and MAMaL$\mathrm{GaM}$. It can be clearly seen that in the clinically-acceptable corner, the solutions of MO-RV-GOMEA (Pareto-)dominate the solutions of both NSGA-II and MAMaLGaM, suggesting that exploiting the information of linkages between problem variables (i.e., dwell 
Efficient, Effective, and Insightful Tackling of the High-Dose-Rate Brachytherapy Treatment Planning Problem for Prostate Cancer using Evolutionary Multi-Objective Optimization Algorithms

GECCO '17 Companion, July 15-19, 2017, Berlin, Germany
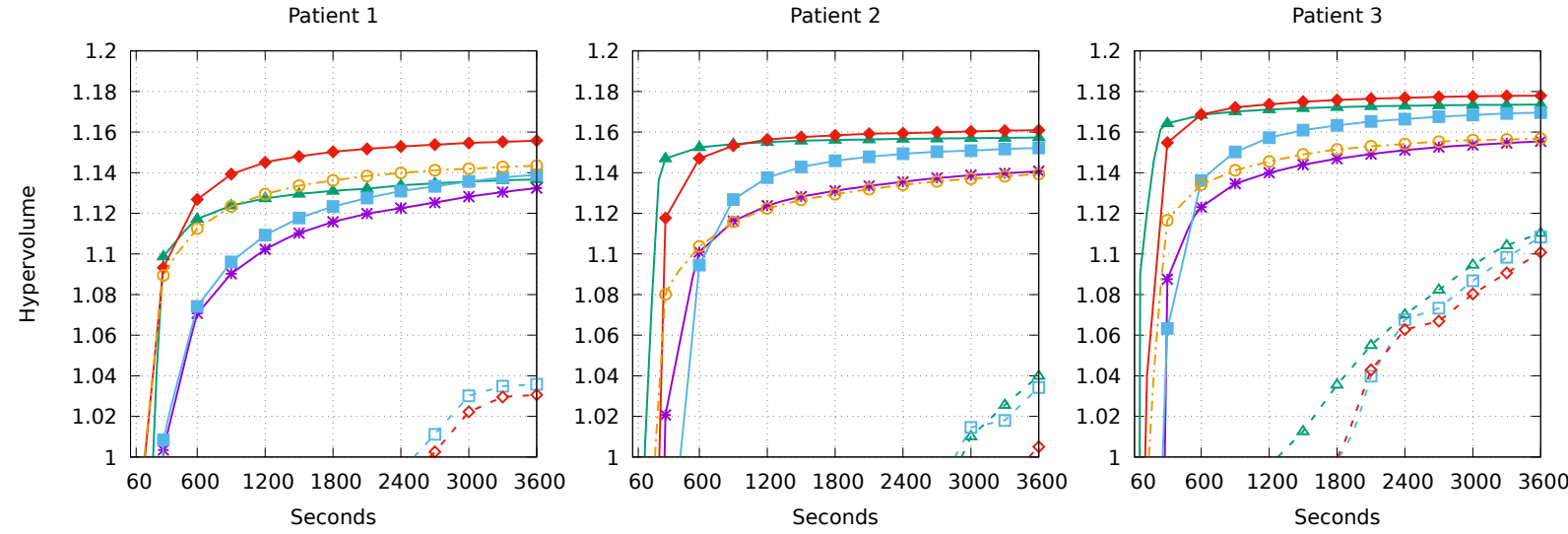

MO-RV-GOMEA UF (full) $-\bar{\Delta}-$

MO-RV-GOMEA LT

MO-RV-GOMEA LT (full) - - -

MO-RV-GOMEA fixed LT $\longrightarrow$

MO-RV-GOMEA fixed LT (full) - $\diamond$

NSGA-II $\rightarrow$

Figure 1: The average hypervolume values of the Pareto fronts of optimization algorithms over time.
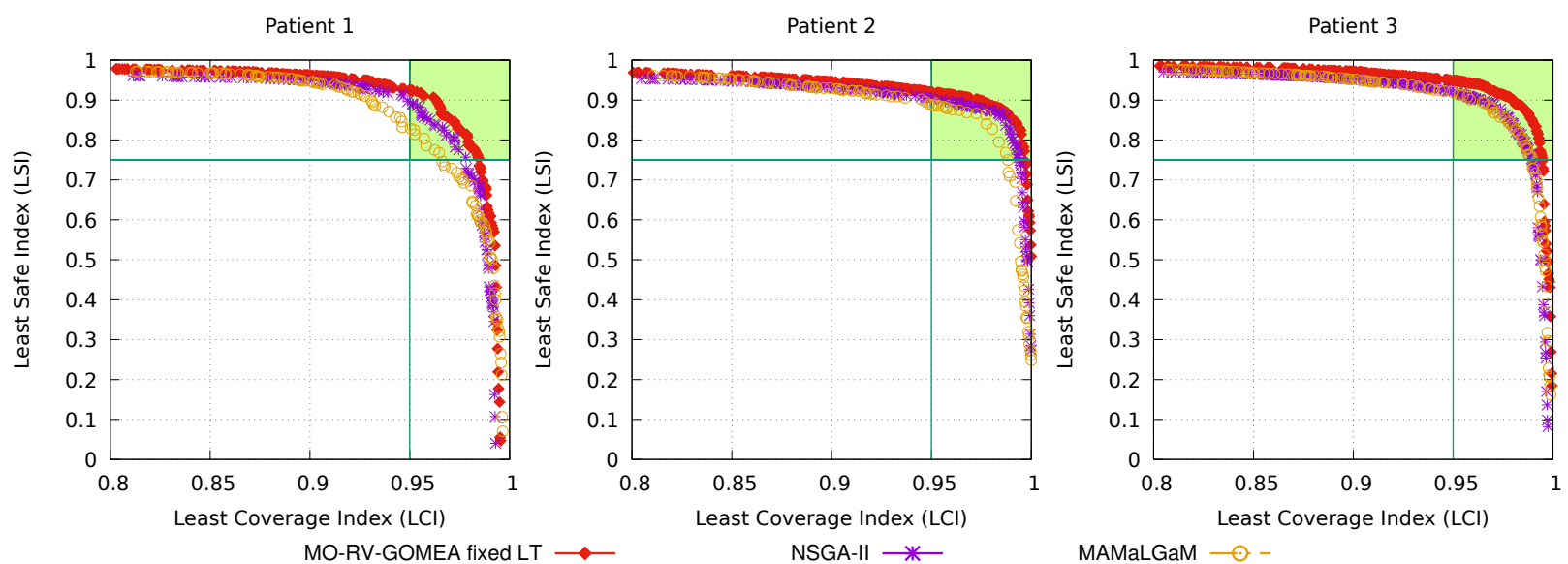

MAMaLGaM $\bigcirc-$

Figure 2: Pareto fronts combined from 30 optimization runs of each algorithm after running for 1 hour.

times) benefits the optimization algorithm in reaching treatment plans of higher quality.

Since the treatment planning process in practice is highly timeconstrained, which should not take more than 1 hour, it is interesting to investigate the results of optimization algorithms with a shorter time span. Figure 3 shows the Pareto fronts combined from 30 optimization runs of each algorithm after running for $10 \mathrm{~min}$ utes. It can be seen that, in all three cases, MO-RV-GOMEA can obtain Pareto fronts of high-quality treatment plans much faster than NSGA-II and MAMaLGaM. Especially in the clinically-acceptable corner, the treatment plans found by MO-RV-GOMEA clearly dominate the results of NSGA-II and MAMaLGaM. Comparing Figure 2 and Figure 3, the improvements of the Pareto fronts of MO-RVGOMEA are not as substantial as the improvements of the Pareto fronts of NSGA-II and MAMaLGaM obtained by an extra 50 minute runtime. This suggests that MO-RV-GOMEA can simply be run in only 10 minutes to achieve the results of the other MOEAs running for 1 hour. Overall, the results appear very promising. As a first next step, these sets of treatment plans exhibiting natural tradeoffs in BT treatment planning will be analyzed with clinical experts and rigorously compared with clinically-approved treatment plans.

\section{CONCLUSION}

In this paper, we presented how the BT treament planning problem can be formulated and tackled by a novel multi-objective optimization approach in which only two objectives are considered, rather than having a single objective for each clinical objective that is typically found in clinical requirements. In particular, those clinical objectives that express to maximize irradiation are grouped in one objective, as are those that express to minimize irradiation. By carefully remapping these clinical objectives and using the worst one in each group as the value in the bi-objective optimization function, a risk-averse, guaranteed minimal performance model is obtained such that the results of which are straightforward to interpret. We 


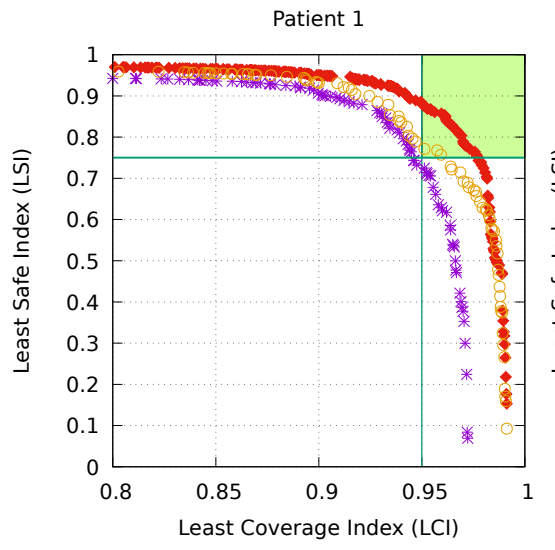

MO-RV-GOMEA fixed LT —

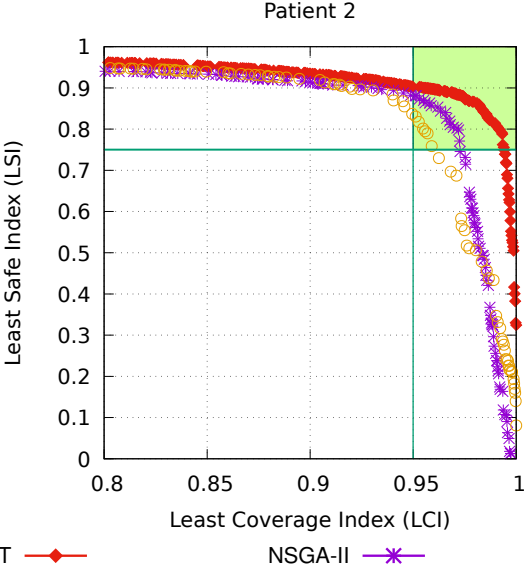

Patient 2

NSGA-II $\rightarrow$

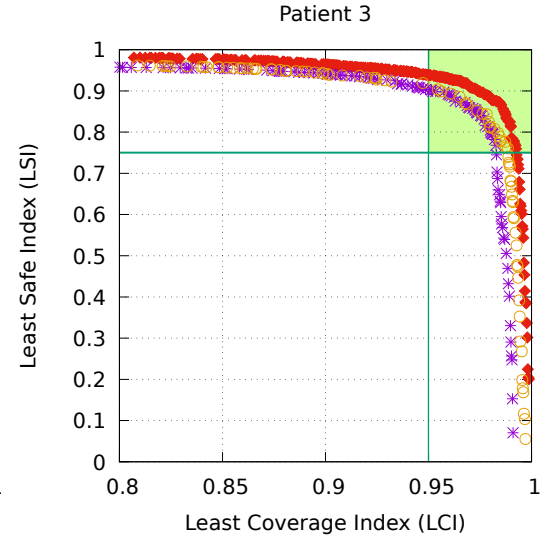

MAMaLGaM $\bigcirc-$

Figure 3: Pareto fronts combined from 30 optimization runs of each algorithm after running for 10 minutes.

proposed that the recently-introduced MO-RV-GOMEA, a promising optimizer that can exploit hierarchical linkages between problem variables, is an especially promising optimizer to tackle the problem because MO-RV-GOMEA allows partial evaluations to be carried out when existing solutions are only locally altered at a few variables, we suggested that this feature can be exploited using the dependencies between dwell positions and their geometry information. Using linkage exploitation and partial evaluations, MO-RV-GOMEA is capable of outperforming both the widely-used MOEA NSGA-II and the state-of-the-art MOEDA MAMaLGaM in obtaining a set of high-quality treatment plans in a $1 / 6$ fraction of the planning time budget. This, together with the fact that Pareto fronts are obtained that insightfully visualize the main trade-off in BT treatment planning, leads us to conclude that MO-RV-GOMEA is a very promising algorithm to develop and use further for the automated design of BT treatment plans.

\section{ACKNOWLEDGMENTS}

This work is part of the research programme IPPSI-TA with project number 628.006.003, which is financed by the Netherlands Organisation for Scientific Research (NWO) and Elekta. The authors further acknowledge the financial support provided by the Maurits en Anna de Kock Stichting for a high-performance computing system. The authors would like to thank Dr. Niek van Wieringen (Department of Radiation Oncology, Academic Medical Center, Amsterdam, The Netherlands) for his support in providing the patient data and Dr. Rob van der Laarse (Quality Radiation Therapy B.V., Zeist, The Netherlands) for his advice on radiation dose calculation.

\section{REFERENCES}

[1] P. A. N. Bosman. 2010. The anticipated mean shift and cluster registration in mixture-based EDAs for multi-objective optimization. In Genetic and Evolutionary Computation Conference, GECCO 2010, Proceedings, Portland, Oregon, USA, Fuly 7-11, 2010. 351-358. DOI : http://dx.doi.org/10.1145/1830483.1830549

[2] A. Bouter, N. H. Luong, C. Witteveen, T. Alderliesten, and P. A. N. Bosman. 2017 The Multi-Objective Real-Valued Gene-Pool Optimal Mixing Evolutionary Algorithm. In Genetic and Evolutionary Computation Conference, GECCO '17, Berlin Germany, fuly 15-19, 2017.

[3] K. Deb. 2001. Multi-Objective Optimization Using Evolutionary Algorithms. John Wiley \& Sons, Inc. http://dl.acm.org/citation.cfm?id=559152
[4] K. Deb, A. Pratap, S. Agarwal, and T. Meyarivan. 2002. A fast and elitist multiobjective genetic algorithm: NSGA-II. IEEE Transactions on Evolutionary Computation 6, 2 (Apr 2002), 182-197. DOI : http://dx.doi.org/10.1109/4235.996017

[5] A. M. Dinkla, R. van der Laarse, E. Kaljouw, Bradley R. P., K. Koedooder, N. van Wieringen, and A. Bel. 2015. A comparison of inverse optimization algorithms for $\mathrm{HDR} / \mathrm{PDR}$ prostate brachytherapy treatment planning. Brachytherapy 14, 2 (2015), 279-288. DOI : http://dx.doi.org/10.1016/j.brachy.2014.09.006

[6] P. J. Hoskin, A. Colombo, A. Henry, P. Niehoff, T. P. Hellebust, F. A. Siebert, and G. Kovacs. 2013. GEC/ESTRO recommendations on high dose rate afterloading brachytherapy for localised prostate cancer: An update. Radiotherapy and Oncology 107, 3 (2013), 325-332. DOI : http://dx.doi.org/10.1016/j.radonc.2013.05.002

[7] A. Karabis, S. Giannouli, and D. Baltas. 2005. 40 HIPO: A hybrid inverse treatment planning optimization algorithm in HDR brachytherapy. Radiotherapy and Oncology 76 (2005), S29. DOI : http://dx.doi.org/10.1016/S0167-8140(05)81018-7

[8] A. Kraskov, H. Stögbauer, and P. Grassberger. 2004. Estimating mutual information. Physical Review E 69 (Jun 2004), 066138. Issue 6. DOI: http://dx.doi.org/10.1103/PhysRevE.69.066138

[9] M. Lahanas, D. Baltas, S. Giannouli, N. Milickovic, and N. Zamboglou. 2000. Generation of uniformly distributed dose points for anatomy-based threedimensional dose optimization methods in brachytherapy. Medical Physics 27, 5 (2000), 1034-1046. DOI : http://dx.doi.org/10.1118/1.598970

[10] E. Lessard and J. Pouliot. 2001. Inverse planning anatomy-based dose optimization for HDR-brachytherapy of the prostate using fast simulated annealing algorithm and dedicated objective function. Medical Physics 28, 5 (2001), 773-779. DOI : http://dx.doi.org/10.1118/1.1368127

[11] N. H. Luong and P. A. N. Bosman. 2012. Elitist Archiving for MultiObjective Evolutionary Algorithms: To Adapt or Not to Adapt. In Parallel Problem Solving from Nature - PPSN XII - 12th International Conference, Taormina, Italy, September 1-5, 2012, Proceedings, Part II. 72-81. DOI: http://dx.doi.org/10.1007/978-3-642-32964-7_8

[12] R. Nath, L. L. Anderson, G. Luxton, K. A. Weaver, J. F. Williamson, and A. S. Meigooni. 1995. Dosimetry of interstitial brachytherapy sources: Recommendations of the AAPM Radiation Therapy Committee Task Group No. 43. Medical Physics 22, 2 (1995), 209-234. DOI : http://dx.doi.org/10.1118/1.597458

[13] D. Thierens and P. A. N. Bosman. 2011. Optimal Mixing Evolutionary Algorithms. In Proceedings of the 13th Annual Conference on Genetic and Evolutionary Computation (GECCO '11). ACM, 617-624. DOI: http://dx.doi.org/10.1145/2001576.2001661

[14] J. L. M. Venselaar, D. Baltas, P. Hoskin, and A. S. Meigooni. 2012. Introduction and Innovations in Brachytherapy. In Comprehensive Brachytherapy. Taylor \& Francis, 3-8. DOI : http://dx.doi.org/doi:10.1201/b13075-3

[15] E. Zitzler and L. Thiele. 1998. Multiobjective optimization using evolutionary algorithms - A comparative case study. In Parallel Problem Solving from Nature PPSN V: 5th International Conference Amsterdam, The Netherlands September 27-30, 1998 Proceedings, A. E. Eiben, T. Bäck, M. Schoenauer, and H. P. Schwefel (Eds.). Springer Berlin Heidelberg, 292-301. DOI : http://dx.doi.org/10.1007/BFb0056872 